\title{
智能变电站运行维护管理浅析
}

\author{
王海超 \\ 国网河南省电力公司镇平县供电公司 \\ DOI:10.32629/hwr.v3i4.2093
}

[摘 要] 当前, 社会生活和生产需要大量电力能源的支撑, 智能变电站也成为了供电中非常关键的场所。为了确保智能变电站 的运行质量,应正确认识智能变电站维护管理中的不足,并采取有效的应对策略,完善智能变电站的运行维护管理。

[关键词]智能变电站; 维护管理; 应当策略

我国电网建设和发展过程中, 信息化、高电化和互动化 已经成为了其主要发展方向。智能电网在电网建设中逐渐成 为了人们关注焦点, 智能变电站能够促进电能转换和转移。合 理应用智能组件技术、光纤技术及传感技术可有效带动我国 智能电网的建设。

\section{1 智能化变电站的结构}

智能变电站在运行的过程中应用多种智能设备保证变 电站数字化建设、信息共享以及平台的信息化及网络化建 设。智能设备一方面提高了信息采集和保护的质量, 另一方 面也可在数据分析的基础上评估变电站内多种设备的运行 状态。智能化变电站建设规模的不断扩大实现了电网的自动 监控、智能调节和线上决策。

智能变电站结构主要由三个层次组成, 分别为分站控制 层、间隔层和过程层。在以上三个部分当中, 过程层通常由 智能化变电站中的一次设备及监控装置组成。站控层在智能 变电站当中是最高级的管理层, 层中涵盖了若干高级管理系 统, 如事件通信系统和对时系统、站域控制系统等。站控层 主要利用智能变电站中的多种设备数据监控及信息交换来 采集数据、操作闭锁、电能量及同步向量。另外, 其还可实 现保护信息的有效管理。

间隔层主要位于过程层和站控层的中间位置, 是一种十 分重要的二次设备, 其通常由继电保护装置、监测功能组、 故障录波器和安全稳定装置等设备构成。间隔层可密切过程 层和站控层之间的联系, 在单位间隔时间中采集到的数据能 够合理地作用在一次设备当中, 从而充分发挥出其在保护方 面的功能。

\section{2 智能变电站的优势}

2.1 减少资源消耗, 保护生态环境

智能变电站建设施工中主要采用光纤电缆器、充油式互 感器和高集成度低消耗的电子元件, 电子设备的更新与接线 的方式在不断完善, 其有效控制了能量消耗, 既降低了工程 建设的成本, 减少了能源消耗, 也减少了变电站内所产生的 电磁和辐射等多种污染, 进而保证了人们身体健康, 达到了 环保的目的。

\section{2 供电可靠性较强}

如今日常供电的可靠性与安全性成为了人们关注的焦
点。智能变电站运行的过程中合理应用先进的设备和专业技 术, 有效保证了设备运行的安全性及可靠性, 同时智能变电 站还可在日常工作中自动完成设备检测和故障分析工作, 第 一时间解决设备故障, 确保变电站安全平稳运行。

\section{3 有效的交互性}

变电站的正常运行保障了正常的发电、变电及电力输送, 对各个环节的交互功能要求比较高。智能变电站更有效的进 行电网运行中的数据统计, 保证信息的准确性以及电网运行 的安全性。智能变电站通过对采集的信息和数据的分析后, 不仅可以进行内部的共享, 还可以将其和网内更高级更复杂 的系统之间进行互动, 确保电网稳定安全的运行。

\section{3 智能化电站运行维护管理中的问题}

3.1 设备内部问题

3. 1.1 快速保护

智能变电站传输的速度要比普通变电站传输的速度慢, 出现这种现象主要是由于在电能传输的过程中, 其流程具有 较强的复杂性, 智能变电站应用电子传感技术, 保证了信息 传输与交换的质量和效率。但是在电能传输的过程中也要合 理应用不同工具予以有效的辅助, 一方面需交换机完成数据 交换, 另一方面还要进行更加复杂的单元合并处理, 其需要 消耗更多的时间来完成综合处理, 工作效率受到了较大的影 响。另外, 在变电站运行的过程中还需要在跳闸口的位置对 信号进行综合处理, 而这也对系统的运行性能产生了较大的 负面影响。

\section{1 .2 设备运行稳定性}

智能变电站传输电能中通信模式也发生了较大的变化, 其主要采用对等通信模式, 这种模式对安全性的要求更高, 操作的流程更多, 且复杂性也明显提高。由于传输的过程中 主要利用软件实现装置隔离, 因此若出现软件故障, 则系统 就会无法正常运行。与普通的变电站相比, 其风险更大, 稳定 性较低。所以, 为了减少由于系统运行稳定性造成的损失, 智能变电站在日常运行的过程中也应积极地保护和维修多 种软件, 促进系统稳定运行。

\section{1 .3 设备可靠性}

智能变电站运行中合理采用光纤技术有效提高了传输 效率, 但是也影响了设备的可靠性。光学互感器的敏感度较 
高, 光纤和玻璃存在着十分明显的矛盾, 极易受到多种因素 的影响。若互感器受到较大的影响, 就会影响其作用与性能, 严重时还会导致系统故障。此外, 电子互感器组装的过程中 需要借助多种元件和模块。元件运行的过程中也需要电力的 支撑, 所以在系统运行的过程中, 需要消耗较多的电能, 而这 明显不利于提高电能的应用效率。

再者, 电力供应不足还会破坏系统运行的稳定性。结合 电磁理论, 磁场会阻碍电子运行, 进而对电子的工作路径产 生较大的影响, 降低了信号质量, 破坏了设备运行的稳定性, 最终造成不同程度的损失, 不利于提升系统的可靠性。

3.2 信息保密较差, 信息传递中面临着较大的风险

智能变电站在运行的过程中主要采用对等信号完成数 据传输。智能设备运行中会产生数据信号, 之后可利用设备 输出信号, 在局域网的支持下分析输出的信号数据, 确保系 统的安全运行。该通讯模式也存在着十分明显的不足, 信号 传输中安全防护性能较差, 如设备在运行的过程中受到干扰 和攻击, 则自动化系统将无法完成信息传递。所以, 必须设置 相应的软件设备来确保信息的安全稳定传递。

3.3 压板操作环境设备运行中出现的问题

智能变电站压板维护中, 应高度重视原始设备的状态控 制。因为设备运行参数无法更改, 如出现压板错误, 则智能变 电系统维护处理无法顺利开展, 只能采取多种措施来解决措 施。再者, 变电站智能信息系统运行的过程中需要收集、传 输和分析信息, 传输的过程中需确保设备运行中的电能供应, 长期的电能供应会增大设备的温度, 长此以往就会明显缩短 设备的使用寿命, 加速设备老化。

\section{4 智能变电站运行维护管理的策略}

4. 1 高度重视智能变电站设备的管理与维护

智能变电站运行的安全与稳定与变电站内设备的质量 和性能有着十分密切的联系。所以要采取多种措施完善智能 变电站设备维护管理。在设备运行管理的过程中, 变电站管 理单位需指派专业的技术人员定期检查巡视设备的运行状 态和安全性。针对电子传感器等关键设备, 需时常调试与检 查, 若在检查的过程中发现问题, 则需及时更换全新的设备。 在设备的运行管理中, 应当采取多种有效的措施不断优化变 电站操作人员的技术水平。且积极组织操作人员和技术人员 参与通讯技术专业知识培训, 让所有的技术人员能够严格按 照规范的要求操控智能终端、操控接口以及继电保护器等重 要设备, 若出现突发问题, 也可对其予以科学处理。

\section{2 加强智能组件的维护管理}

智能变电站运行的过程中, 智能组件的负荷所占比重较 大。智能变电站维护管理的过程中, 智能组件维护管理占据 着十分重要的位置。若要促进智能变电站的平稳运行, 就应 采取有效措施不断优化和完善顺序操控。所以, 在智能组件 维护管理中, 相关人员需采取有效措施不断增强操控的科 学性与准确性, 全面结合规范的要求和标准的基本内容, 规 范操作, 从而加强监控的有效性, 确保智能组件的安全平稳 运行。

4.3 完善智能变电站的安全防护

智能变电站建设和运行的过程中, 需要根据智能变电站 的实际情况, 采取科学有效的安全防护措施, 督促工作人员 做好定期的巡视和检查工作, 第一时间发现故障, 且采取有 效的处理措施。在变电站建设中, 需要应用电流电压互感器 调节和控制采样电缆, 有效减少中间环节, 不断增强变电站 运行的安全性和可靠性。此外, 在采取安全防护措施的过程 中, 要先对设备予以全面检查, 保证设备仪表等参数均在合 理的范围内。且仔细检查智能终端、自动装置及保护装置的 连接情况, 明确设备的使用温度是否处于正常范围。

4.4 对压板和交换机进行有效管理

智能变电站运行系统中需要在信息化数字平台实现切 区、定值的判断与规划。数字化切板能够保护出口跳压板, 保证硬压板能够正常运行。禁止停役设备的检修状态, 增加 LED 软压板功能, 保证设备远端的固定值, 确定软压板的基本 可靠性。

\section{4 结束语}

综合以上分析我们可以获知, 就我国的电网发展而言, 建设好智能变电站是非常重要且关键的, 这不但可以有效的 节能工程成本, 同时还能提升工程的经济效益。为此, 我们就 应构建科学的监管制度, 并通过培训等方式不断提升技术 人员的专业技能, 进而最大程度的保障智能变电站的安全 运行。

[参考文献]

[1]陈向东,张杰.浅析智能变电站运维管理存在的问题 及改进建议[J].城市建设理论研究(电子版),2018(14):54-55.

[2]王超,刘琪.智能变电站运行维护管理措施探析[J].中 国战略新兴产业,2018(36):249-250.

[3]乔宇,李鹏,秦浩.智能变电站运行维护工作中存在的 问题及应对措施[J].山东工业技术,2018(13):151. 